\title{
Análisis de los factores determinantes del uso de mensajes SMS para participar en programas de televisión
}

\section{Analysis of key factors influencing SMS usage to participate in television programmes}

\author{
Carla Ruiz Mafé1 / Silvia Sanz Blas / Juan Fernando Tavera $²$ \\ Recibido el 23 de abril de 2009, , aceptado el 6 de julio de 2010 \\ $\mathrm{N}^{\mathrm{o}}$ de clasificación JEL: M31, M37. \\ DOI: $10.5295 / \mathrm{cdg} .100157 \mathrm{cr}$ \\ Reseña Bibliográfica: RUIZ MAFÉ, C.; SANZ BLAS, S.; FERNANDO TAVERA, J. (2010): “Análisis \\ de los factores determinantes del uso de mensajes SMS para participar en programas de televisión"; \\ Cuadernos de Gestión, Vol. 10. № 2, pp. 117-132. DOI: 10.5295/cdg.100157cr
}

\section{Resumen:}

El envío de mensajes cortos a programas, anuncios o concursos de televisión se ha convertido hoy en día en una fuente importante de ingresos tanto para los operadores de telefonía móvil como para las cadenas y productoras televisivas. El objetivo del presente trabajo es analizar cómo contribuye la actitud y la relación individuomedio en que el telespectador utilice este servicio de mensajería para participar en los programas televisivos. Los resultados ponen de relieve que tanto la compatibilidad del individuo con el servicio como el entretenimiento percibido al participar en este tipo de programas y la actitud hacia el uso son factores determinantes de la utilización del servicio.

Palabras clave:

Móvil, Televisión, compatibilidad, entretenimiento, actitud.

\section{Abstract:}

SMS sent in response to programmes, advertising and television competitions are currently an important source of income for mobile phone operators, television chains and producers. This present work aims to analyse the role of the attitude and individual-media variables which encourage televiewers to use this messaging service to participate in television programmes. The study was carried out on a sample of 205 SMS users. The results show that individual compatibility with the service, the perceived entertainment value of participating in this type of programme and attitude towards use are determinants of SMS use.

\section{Keywords:}

Mobile, television, compatibility, enjoyment, attitude.

\footnotetext{
${ }^{1}$ La autora agradece la financiación del proyecto UV-AE-10-24521 concedido por el Vicerrectorado de Investigación de la Universidad de Valencia

${ }^{2}$ Departamento de Comercialización e Investigación de Mercados. Facultad de Economía, Universidad de Valencia. Avda. Tarongers s/n. 46022. Valencia-ESPAÑA. carla.ruiz@uv.es; silvia.sanz@uv.es Facultad de Ciencias Económicas, Universidad de Antioquia. Calle 67 No. 53-108. Bloque 13. Oficina 405. Medellin - COLOMBIA.jftavera@economicas.udea.edu.co
} 


\section{INTRODUCCIÓN}

Para los operadores móviles, dadas las elevadas tasas de penetración que presenta hoy en día la telefonía móvil en España (120\%) (Netsize, 2010), resulta cada vez más difícil conseguir nuevos clientes, lo que conlleva, que para poder incrementar sus beneficios, tengan que desarrollar nuevos servicios y aplicaciones compatibles con su tecnología o buscar un uso más intenso de los mismos. Uno de los servicios que mayor aceptación está teniendo en estos últimos años es el envío de mensajes cortos, más conocidos como SMS (Munnukka, 2007), que en España generó en el último trimestre de 2007, 2.628 millones de euros (Comisión del Mercado de Telecomunicaciones, 2008). Durante el año 2008 el mercado de SMS ha continuado incrementando el número de suscriptores, posiblemente debido a que los mensajes cortos son la forma más sencilla, económica y rápida de comunicación entre amigos y entre usuarios y empresas.

La importancia que está adquiriendo este servicio lleva a su uso con finalidades de marketing, no solo a través del propio terminal móvil sino también a través de otros medios de comunicación como la televisión, que presenta una mayor interacción mediante mensajes de texto (Beyer et al., 2007; Herring y Zelenkauskaite, 2009). Hoy en día, la interacción del individuo con la televisión a través de mensajes SMS incluye la participación en programas, concursos o anuncios publicitarios con el fin de realizar votaciones, opinar apareciendo en pantalla, descargar contenidos, concursar en sorteos o juegos, comprar una melodía y hasta chatear a través de la pantalla de televisión en algunos formatos (Beyer et al., 2007). Esta revolucionaria tecnología hace posible que la audiencia actúe de forma instantánea sobre un programa/concurso de televisión, utilizando su teléfono móvil como si de un mando a distancia se tratase.

De este modo, el envío de mensajes SMS a programas, anuncios y concursos televisivos constituye una fuente de ingresos cada vez más significativa tanto para los operadores de telefonía móvil, que ven intensificado el uso del servicio, como para las productoras televisivas, que lo utilizan como estrategia promocional para conseguir captar la atención del individuo y que el mismo se convierta en telespectador activo del programa.

Pero no todos los consumidores están realizando un uso intensivo de este servicio, por tanto resulta de interés analizar cómo influye la actitud y la interacción individuo-medio en la aceptación de este servicio. La revisión de la literatura pone de relieve que la actitud hacia el uso es un factor clave en el análisis del comportamiento de adopción de la tecnología (Davis, 1989; Davis et al., 1989; Kuo y Yeng, 2009; Liao et al., 2007; Lu et al., 2009). Junto a la actitud, otros factores pueden influir en la decisión de utilizar los servicios prestados a través del móvil. Entre ellos, cabe destacar las motivaciones de carácter hedónico (Liao et al., 2007; Lu et al., 2009; Nysveen et al., 2005a,b) y la compatibilidad percibida con el servicio (Mallat et al., 2009).

Por lo anteriormente expuesto, el objetivo del presente trabajo es analizar la influencia de las actitudes hacia el uso, entretenimiento percibido y compatibilidad con el servicio en la intención de uso de mensajes SMS para participar en programas televisivos. 


\section{REVISIÓN DE LA LITERATURA}

\subsection{Marco Conceptual}

\section{Actitud}

La actitud es una evaluación positiva o negativa del individuo acerca de un determinado objeto o comportamiento (Ajzen, 1991; Fishben y Ajzen, 1975) e incorpora sentimientos o respuestas afectivas hacia los mismos. Hace referencia a la predisposición global de un individuo hacia el desarrollo de una conducta. Esta actitud es el resultado de las creencias de los sujetos respecto al comportamiento y sus resultados y de la importancia que se dé a dichas creencias.

La literatura sobre psicología social, sugiere claramente que la actitud tiene dos componentes: el afectivo y el cognitivo (Bagozzi y Burnkrant, 1979, 1985; Chaiken y Stangor, 1987; McGuire, 1985; Weiss y Cropanzano, 1996). El componente afectivo de la actitud hace referencia a cuánto le gusta a una persona el objeto de sus pensamientos (McGuire, 1985) y mide el grado de atracción emocional hacia el objeto (Bagozzi y Burnkrant, 1979). Por otro lado, el componente cognitivo hace referencia a las creencias concretas de un individuo con relación al objeto (Bagozzi y Burnkrant, 1979, 1985) y consiste en la evaluación, juicio, recepción o percepción del objeto de pensamiento basado en valores (Chaiken y Stangor, 1987). En particular, la dimensión cognitiva de la actitud influye directamente sobre el uso de los sistemas de información del individuo, mientras que la dimensión afectiva necesita ser tratada como una variable resultado en si misma.

Si nos centramos en el ámbito de nuestro estudio (SMS), la actitud podría entenderse como "la predisposición favorable o desfavorable del individuo hacia el uso de SMS para participar en programas de televisión". Las actitudes hacia el móvil no sólo son el resultado de las creencias respecto al comportamiento sino que también vienen determinadas por otras variables sobre la relación individuo-medio, como la compatibilidad y el entretenimiento percibido del programa (Baron, 2006; Nysveen et al., 2005 a,b).

\section{Compatibilidad}

En el marco de la Teoría de Difusión de Innovaciones, la compatibilidad ha sido definida como el grado en que una innovación es consistente con los patrones de comportamiento, estilos de vida, necesidades y valores de los adoptantes (Rogers, 1995). De acuerdo con Laforet y Li (2005), los consumidores utilizan las tecnologías móviles debido a que les permiten cubrir necesidades específicas, como el acceso a los servicios ofertados desde cualquier lugar público o privado mientras se están desplazando de un sitio a otro.

El envío de SMS para participar en programas de Televisión puede considerarse una innovación debido a que representa un cambio respecto a participar utilizando otros canales de comunicación como el correo postal o una llamada telefónica, y permite a los consumidores utilizarlo independientemente de su ubicación. En el contexto analizado, la compatibilidad se define como "el grado en que un consumidor considera que enviar un 
SMS para participar en un programa de televisión encaja con su estilo de vida, necesidades y con la forma en que suele participar en este tipo de programas".

\section{Entretenimiento percibido}

La Teoría de los Usos y Gratificaciones (Katz et al., 1974; McQuail, 1995) ha sido considerada una de las teorías más influyentes en el estudio de los medios de comunicación masivos ya que, a diferencia de otros enfoques o teorías, ha permitido comprender mejor qué necesidades cubre el contenido de la comunicación y las gratificaciones aportadas a los públicos de los medios de masas. De este modo, permite responder a la pregunta de para qué usa el consumidor el medio (McQuail, 1995) y, por tanto, qué necesidades o deseos es capaz de cubrir (Anderson y Meyer, 1975). Esta teoría postula que los individuos aceptan e interactúan diariamente con los medios no solamente por motivos extrínsecos sino también intrínsecos.

Los motivos intrínsecos hacen referencia a gratificaciones internas, es decir, emociones, diversión y socialización con otros consumidores (Babin et al., 1994) mientras utilizan los medios. Los individuos guiados por motivaciones intrínsecas cuando utilizan el móvil buscan valores subjetivos y personales que no están asociados al desempeño de una tarea específica. En la actualidad, el móvil está incrementando su valor intrínseco de diversión a través de la participación en juegos o concursos interactivos que son utilizados como herramienta promocional en las compras.

Una de las motivaciones intrínsecas que ha adquirido una importancia notable desde el punto de vista de la investigación es el entretenimiento percibido, que se define como el grado en que una actividad se percibe como disfrutable en sí, más allá de las consecuencias o recompensas que pueden ser anticipadas (Davis et al., 1992). En el contexto objeto de estudio podría definirse como "el grado en que un consumidor considera que los programas de televisión que permiten el envío de SMS son entretenidos y divertidos, más allá de las consecuencias o recompensas que pueden ser anticipadas".

\subsection{Planteamiento de hipótesis}

Diversas investigaciones respaldan la existencia de una relación significativa entre las actitudes y la intención de uso de los servicios del móvil (Kuo y Yeng, 2009; Liao et al., 2007; Lu et al., 2009). Nysveen et al. (2005b) sostienen que la relación entre las actitudes y la intención de uso depende de la categoría de servicio analizado. Así, la intención de utilizar un nuevo servicio se ve más influida por las actitudes que por la experiencia de uso, mientras que en el caso de servicios ya implantados en el mercado son las motivaciones utilitaristas y la experiencia los principales antecedentes de la intención de uso.

El envío de SMS para participar en programas de televisión constituye una innovación. Con el fin de complementar la revisión de la literatura, se plantea como hipótesis a contrastar que actitudes favorables hacia el envío de SMS para participar en programas de televisión deben influir positivamente en la intención de uso de este servicio. 
H1: La actitud influye positivamente en la intención de participar en programas de televisión, mediante el envío de mensajes SMS.

Investigaciones previas (Moore y Benbasat, 1991; Taylor y Todd, 1995) han puesto de manifiesto la influencia directa de la compatibilidad en la actitud del consumidor. Posteriormente, los trabajos de Vijayarasathy (2004) y Chen y Gongmin (2007) evidencian que la compatibilidad influye de forma positiva en la actitud hacia las compras online.

Con el fin de complementar la revisión de la literatura, parece lógico plantear que si la participación en programas de televisión mediante SMS se percibe como compatible con el estilo de vida del usuario del móvil y con la forma en la que éste utiliza los servicios del móvil, se inducirá una actitud favorable hacia el uso de SMS para participar en programas de televisión.

H2: La compatibilidad con el servicio influye positivamente en la actitud hacia el uso de SMS para participar en programas de televisión.

Estudios previos (Lu et al., 2009; Nysveen et al., 2005b) ponen de manifiesto que el entretenimiento percibido influye positivamente en la actitud hacia los servicios del móvil, siendo estas relaciones más intensas en los servicios hedónicos (Nysveen et al., 2005b). En los estudios de Nysveen et al. (2005a, b) se pone de manifiesto la influencia del entretenimiento percibido en la actitud de los hombres hacia el uso de servicios de chat por móvil. Ha et al. (2007) también sostienen que los juegos interactivos de los dispositivos móviles proporcionan entretenimiento derivado de su uso y que a su vez el entretenimiento percibido influye en la actitud hacia su uso.

Con el fin de complementar los hallazgos de investigaciones previas, en la presente investigación se plantea la influencia del entretenimiento percibido como antecedente de la actitud en la siguiente hipótesis:

H3: El entretenimiento percibido al participar en programas de televisión mediante el envio de SMS, influye favorablemente en la actitud del consumidor hacia el uso de SMS para participar en programas de televisión.

El entretenimiento experimentado durante la experiencia de uso del servicio no sólo induce a una actitud favorable hacia el uso, sino que además es capaz de influir en la compatibilidad con el servicio percibida por el individuo (Ha et al., 2007; Nysveen et al., 2005a,b). Por tanto, parece lógico afirmar que aquellos individuos que más disfrutan utilizando el móvil, son los que lo perciben en mayor medida como un instrumento que encaja con su estilo de vida. Por tanto, planteamos la siguiente hipótesis de contraste:

H4: El entretenimiento percibido de los programas de televisión que permiten el envío de SMS, influye favorablemente en la compatibilidad del consumidor con el servicio de SMS para participar en programas de televisión.

En el estudio de Wu y Wang (2005) se evidencia que los individuos que perciben una mayor compatibilidad con el móvil para realizar compras, son los que desarrollan una mayor predisposición hacia la realización en el futuro de comercio electrónico a través del móvil. Posteriormente, Hsu et al. (2007) muestran que la compatibilidad con el uso de Internet móvil influye de forma significativa en la adopción de mensajes multimedia (MMS). En consecuencia, planteamos: 
H5: La compatibilidad con el uso de SMS participar en programas de televisión, influye positivamente en la intención de participar en programas de televisión mediante el envío de SMS.

Actualmente, existe un segmento cada vez mayor de consumidores (especialmente los jóvenes) que utilizan el móvil por motivos intrínsecos, es decir, buscando diversión y salir de la rutina, expresarse, estar a la moda, conseguir un mayor status y sociabilidad.

Basándose en la Teoría de Usos y Gratificaciones, diversos estudios (Leung y Wei, 2000; Nysveen et al., 2005a,b) ponen de manifiesto la influencia de las motivaciones intrínsecas en la decisión de uso de los servicios del móvil.

Por consiguiente, parece lógico plantear que cuanto mayor sea el entretenimiento percibido de un programa en el que se incentive la participación en el mismo a través de SMS, mayor será la intención del consumidor de utilizar este servicio.

H6: El entretenimiento percibido de los programas de televisión que permiten el envío de SMS, influye positivamente en la intención de participar en programas de televisión mediante el envío de SMS.

Las hipótesis planteadas conforman un modelo (ver figura 1) que permite analizar la influencia de la actitud hacia el uso, de la compatibilidad con el servicio y del entretenimiento percibido en la intención de utilizar el servicio de mensajes cortos (SMS) para participar en programas de televisión.

Figura 1

\section{Modelo teórico propuesto}

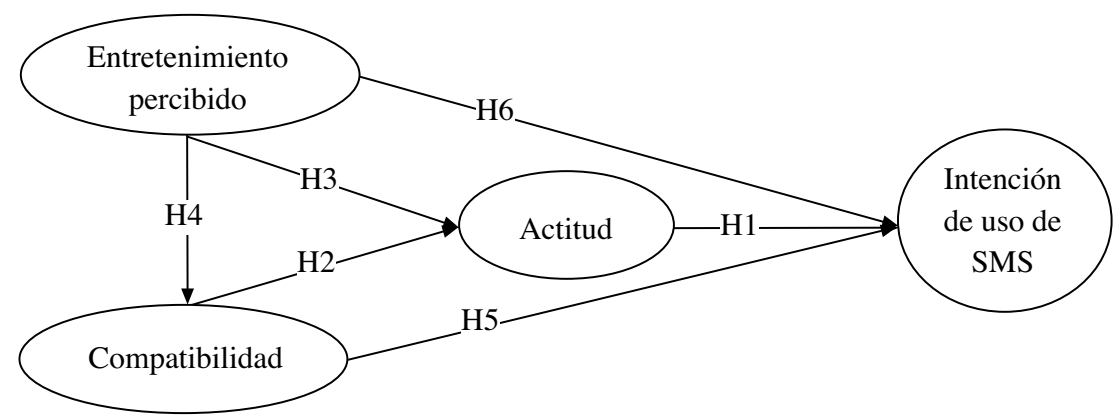

\section{METODOLOGIA}

Los datos objeto de análisis proceden de un estudio de mercado llevado a cabo en Valencia durante el mes de mayo de 2008. La investigación ha sido realizada utilizando una muestra de 205 usuarios del móvil. El método de recogida de información ha sido la entrevista personal con cuestionario estructurado. El procedimiento de muestreo fue por conveniencia, al igual que en numerosos estudios previos realizados en el ámbito de la 
telefonía móvil (Wu y Wang, 2005; Yang, 2005). El cuadro 1 muestra las características principales de la muestra.

Cuadro 1

Características de la muestra

\begin{tabular}{l|c}
\hline VARIABLE & $\mathrm{n}=205$ \\
\hline Género & \\
\hline Hombres & $43,9 \%$ \\
Mujeres & $56,1 \%$ \\
\hline Edad & \\
\hline De 14 a 24 años & \\
De 25 a 34 años & $60,0 \%$ \\
De 35 a 49 años & $31,7 \%$ \\
Más de 50 años & $5,9 \%$ \\
\hline Nivel de estudios & $2,4 \%$ \\
\hline Primer grado (EGB) / Primaria completa & \\
Segundo grado / secundaria / bachiller & \\
Título universitario / técnico / profesional & $2,0 \%$ \\
Posgrado / Master & $32,7 \%$ \\
Doctor / PhD & $47,3 \%$ \\
\hline Nivel de ingresos: "Si los ingresos medios mensuales personales se & $17,1 \%$ \\
sitúan en 1200 euros, su nivel de ingresos es....” & $1,0 \%$ \\
\hline Sin ingresos & \\
Ingresos muy por debajo del promedio & \\
Ingresos debajo del promedio & \\
Ingresos promedio & \\
Ingresos por encima del promedio & \\
Ingresos muy por encima del promedio & \\
\hline
\end{tabular}

Fuente: Elaboración propia.

Dado que son los jóvenes los principales usuarios del teléfono móvil (Bigné et al., 2008; Netsize, 2009), se ha procedido a dividir la muestra en dos grupos (individuos hasta 24 años e individuos de edad superior), con objeto de conocer y analizar sus características más relevantes e identificar diferencias significativas entre ambos grupos (ver Cuadro 2). 


\section{Cuadro 2}

Descripción de la muestra en función de la edad

\begin{tabular}{|c|c|c|c|}
\hline & $\begin{array}{c}\text { Edad } \\
<=24 \text { años }\end{array}$ & $\begin{array}{c}\text { Edad } \\
>24 \text { años }\end{array}$ & \multirow[t]{2}{*}{ Diferencias } \\
\hline VARIABLE & $\mathrm{n}=123$ & $\mathbf{n}=\mathbf{8 2}$ & \\
\hline \multicolumn{4}{|l|}{ Género } \\
\hline $\begin{array}{l}\text { Hombres } \\
\text { Mujeres }\end{array}$ & $\begin{array}{l}41,9 \% \\
58,1 \% \\
\end{array}$ & $\begin{array}{l}52,2 \% \\
47,8 \% \\
\end{array}$ & $\begin{array}{c}X^{2}=18,569 ; \\
0,000\end{array}$ \\
\hline \multicolumn{4}{|l|}{ Nivel de estudios } \\
\hline $\begin{array}{l}\text { Primer grado (EGB) / Primaria completa } \\
\text { Segundo grado / secundaria / bachiller } \\
\text { Título universitario } \\
\text { Posgrado / Master } \\
\text { Doctor / PhD } \\
\end{array}$ & $\begin{array}{c}0 \% \\
48,8 \% \\
43,1 \% \\
8,1 \% \\
0 \% \\
\end{array}$ & $\begin{array}{l}\mathbf{4 , 9 \%} \\
\mathbf{8 , 5 \%} \\
\mathbf{5 3 , 7 \%} \\
\mathbf{3 0 , 5 \%} \\
\mathbf{2 , 4 \%} \\
\end{array}$ & $\begin{array}{c}X^{2}=48,947 \\
0,000\end{array}$ \\
\hline \multicolumn{4}{|l|}{$\begin{array}{l}\text { Nivel de ingresos: "Si los ingresos medios mensua- } \\
\text { les personales se sitúan en } 1200 \text { euros, su nivel de } \\
\text { ingresos es...." }\end{array}$} \\
\hline $\begin{array}{l}\text { Sin ingresos } \\
\text { Ingresos muy por debajo del promedio } \\
\text { Ingresos debajo del promedio } \\
\text { Ingresos promedio } \\
\text { Ingresos por encima del promedio } \\
\text { Ingresos muy por encima del promedio }\end{array}$ & $\begin{array}{c}\mathbf{7 3 , 6 \%} \\
\mathbf{5 , 7 \%} \\
\mathbf{6 , 5 \%} \\
10,6 \% \\
\mathbf{1 , 6 \%} \\
\mathbf{2 , 0 \%} \\
\end{array}$ & $\begin{array}{c}18,3 \% \\
3,7 \% \\
8,5 \% \\
45,1 \% \\
19,5 \% \\
4,9 \% \\
\end{array}$ & $\begin{array}{c}\mathrm{X}^{2}=79,384 \\
\mathbf{0 , 0 0 0}\end{array}$ \\
\hline \multicolumn{4}{|l|}{ Frecuencia de las llamadas } \\
\hline $\begin{array}{l}\text { Más de } 10 \text { veces al día } \\
\text { Entre } 6 \text { y } 10 \text { veces al día } \\
\text { Entre } 1 \text { y } 5 \text { veces al día } \\
\text { Algunas veces en la semana }\end{array}$ & $\begin{array}{l}7,3 \% \\
19,5 \% \\
61,8 \% \\
11,4 \% \\
\end{array}$ & $\begin{array}{l}20,7 \% \\
31,7 \% \\
35,4 \% \\
12,2 \% \\
\end{array}$ & $\begin{array}{c}\mathrm{X}^{2}=20,312 \\
0,000\end{array}$ \\
\hline \multicolumn{4}{|l|}{ Mensajes promedio } \\
\hline $\begin{array}{l}\text { Ninguno } \\
\text { Entre } 1 \text { y } 5 \\
\text { Entre } 6 \text { y } 10 \\
\text { Entre } 11 \text { y } 15 \\
\text { Más de } 15\end{array}$ & $\begin{array}{c}7,3 \% \\
81,4 \% \\
8,9 \% \\
0 \% \\
2,4 \% \\
\end{array}$ & $\begin{array}{c}20,7 \% \\
65,9 \% \\
9,8 \% \\
2,4 \% \\
1,2 \% \\
\end{array}$ & $\begin{array}{c}X^{2}=15,079 \\
0,000\end{array}$ \\
\hline \multicolumn{4}{|l|}{ Motivos de uso SMS (valores medios sobre 5) } \\
\hline $\begin{array}{l}\text { Uso para comunicarse con amigos, familiares o } \\
\text { colegas } \\
\text { Uso para opinar en programas de televisión y } \\
\text { aparecer en pantalla }\end{array}$ & $\begin{array}{l}4,67 \\
3,10\end{array}$ & $\begin{array}{l}3,96 \\
2,00\end{array}$ & $\begin{array}{c}F=22,780 \\
\mathbf{0 , 0 0 0} \\
F=\mathbf{5 , 6 4 9} \\
\mathbf{0 , 0 1 8}\end{array}$ \\
\hline Actitud (valores medios sobre 5) & 3,30 & $\mathbf{3 , 1 7}$ & $F=0,693 ; 0,406$ \\
\hline Compatibilidad (valores medios sobre 5) & 3,79 & 3,25 & $F=6,768 ; 0,010$ \\
\hline Entretenimiento (valores medios sobre 5) & 2,89 & 2,77 & $F=0,677 ; 0,412$ \\
\hline Intención de uso (valores medios sobre 5) & 3,67 & 3,06 & $F=6,375 ; 0,012$ \\
\hline
\end{tabular}


Del cuadro 2 se desprende que los entrevistados muestran una actitud positiva hacia el envío de SMS para participar en programas de televisión, siendo además un servicio compatible con su forma de vida. Dichos programas son considerados entretenidos y divertidos, mostrando el encuestado una intención positiva de participar en los mismos mediante envío de mensajes cortos.

El grupo más joven (de edad similar o por debajo a 24 años) se caracteriza por estar formado por un $41,9 \%$ de hombres y un $58,1 \%$ mujeres, con un nivel de formación media o superior y preferentemente sin ingresos $(73,6 \%)$. En el grupo de edad superior a 24 años existe un ligero predominio de los hombres $(52,2 \%)$, con formación media y/o superior y con un nivel de ingresos similar o por encima de la media $(69,5 \%)$. El test de la chicuadrado revela la existencia de diferencias significativas en todas las variables sociodemográficas analizadas.

La frecuencia diaria de uso del móvil para efectuar llamadas es más elevada en el grupo de edad superior a 24 años (el 52,4\% realizan más de 6 llamadas diarias). Aunque los más jóvenes efectúan menos llamadas, éstas se ven compensadas con un mayor número de mensajes SMS enviados. De ese modo, los más jóvenes utilizan en mayor medida los mensajes cortos para comunicarse con amigos, familiares o conocidos. Nuevamente, el test de la chi-cuadrado revela diferencias significativas.

Son los más jóvenes los que consideran más entretenidos los programas en los que se puede participar con SMS, los que están más de acuerdo en que el envío de SMS para participar en dichos programas es una buena idea, los que consideran que es más compatible con sus estilos de vida y los que muestran una mayor intención de uso futuro.

En el cuadro 3, se describe cómo se ha realizado la medición de las variables utilizadas en el estudio empírico. Todas las variables han sido medidas con escalas Likert de 5 puntos. Para adaptar la actitud al contexto objeto de estudio se han tomado como referencia los estudios de Taylor y Todd (1995) y de George (2004). La intención de uso ha sido medida a partir de los estudios de Taylor y Todd (1995) y de Cheong y Park (2005). Para medir la compatibilidad se ha tomado como referencia el estudio de Wu y Wang (2005) sobre compra por móvil, mientras que el entretenimiento percibido ha sido medido a partir de los estudios de Nysveen et al. (2005a, 2005b).

Cuadro 3

Medición de las variables en el análisis

\begin{tabular}{l|c|l}
\hline \multicolumn{1}{c|}{ CONSTRUCTO } & \multicolumn{1}{c|}{ Medias } & \multicolumn{1}{c}{ FUENTE } \\
\hline \begin{tabular}{l|l} 
Actitud: Enviar mensajes SMS para participar en programas de \\
televisión....
\end{tabular} & & \\
\hline Es una idea que me gusta & 2,93 & Taylor y Todd \\
Me parece una idea inteligente & 3,49 & $(1995 ;$ George \\
Es una buena idea & 3,51 & $(2004)$. \\
Es una experiencia positiva & 2,96 & \\
\hline
\end{tabular}




\begin{tabular}{|c|c|c|}
\hline CONSTRUCTO & Medias & FUENTE \\
\hline $\begin{array}{l}\text { Intención de uso: Con relación al uso de SMS para participar en } \\
\text { programas de Televisión en los próximos } 6 \text { meses... }\end{array}$ & & \multirow[b]{2}{*}{$\begin{array}{l}\text { Adaptado de: } \\
\text { Taylor y Todo } \\
\text { (1995); } \\
\text { Nysveen et al } \\
\text { (2005a) }\end{array}$} \\
\hline $\begin{array}{l}\text { Tengo la intención de participar frecuentemente en programas de } \\
\text { televisión mediante el envío de SMS } \\
\text { Pienso que enviaré mensajes SMS para participar en programas } \\
\text { de televisión } \\
\text { Recomendaré a otros que utilicen los mensajes SMS para partici- } \\
\text { par en los programas-anuncios-concursos de televisión }\end{array}$ & $\begin{array}{l}3,42 \\
3,36\end{array}$ & \\
\hline $\begin{array}{l}\text { Compatibilidad: Enviar mensajes SMS para participar en pro- } \\
\text { gramas de televisión... }\end{array}$ & \multirow[b]{2}{*}{$\begin{array}{l}3,30 \\
3,40 \\
3,93\end{array}$} & \multirow[b]{2}{*}{$\begin{array}{l}\text { Adaptado de: } \\
\text { Wu y Wang } \\
(2005)\end{array}$} \\
\hline $\begin{array}{l}\text { Encaja con mi estilo de vida } \\
\text { Encaja con la forma en la que me gusta participar en programas } \\
\text { de televisión } \\
\text { Es compatible con muchos aspectos relativos a la participación } \\
\text { en programas de televisión }\end{array}$ & & \\
\hline $\begin{array}{l}\text { Entretenimiento percibido: Los programas de televisión que } \\
\text { permiten enviar mensajes SMS }\end{array}$ & & \multirow[b]{2}{*}{$\begin{array}{l}\text { Adaptado de: } \\
\text { Nysveen et al } \\
(2005 a, b)\end{array}$} \\
\hline $\begin{array}{l}\text { Son entretenidos } \\
\text { Son placenteros } \\
\text { Son excitantes } \\
\text { Son divertidos } \\
\text { Disfruto viendo estos programas de televisión }\end{array}$ & $\begin{array}{l}3,05 \\
2,74 \\
2,59 \\
3,07 \\
2,76\end{array}$ & \\
\hline
\end{tabular}

Fuente: Elaboración propia.

\section{RESULTADOS}

\subsection{Análisis de las escalas de medida}

En primer lugar, se comprobó que las escalas de medida utilizadas cumplían las propiedades psicométricas establecidas por la literatura. Para valorar la validez convergente y discriminante se recurrió al análisis factorial confirmatorio, utilizándose para ello el software estadístico EQS versión 6.1 y el método de estimación Máxima Verosimilitud Robusto, ya que este método permite operar con mayor seguridad en muestras que presentan cierto grado de anormalidad de los datos (Jöreskog y Sorbom, 1990).

Para garantizar la validez convergente fueron eliminados aquellos ítems cuyas cargas factoriales, siendo significativas, eran inferiores a 0.60 (Bagozzi y Yi, 1988) o para los que el test de los multiplicadores de Lagrange sugería relaciones significativas sobre un factor distinto a aquel del que eran indicadores (Hatcher, 1994). Tan sólo tuvo que ser eliminado el ítem ACT1 de la escala de actitud. Tras la eliminación del mencionado ítem, todos los coeficientes de regresión factorial resultaron significativos $(t>2.56)$, las cargas estandarizadas superiores a 0.6 , obteniéndose además un buen ajuste del modelo (S-B Chi Square $=245.740$ df. $=104$, p <.05; BBNFI=0.917; BBNNFI=0.957; CFI=0.966; IFI=0.967; RMSEA=0.055). Estos resultados confirmaron la existencia de validez convergente. 
Para valorar la validez discriminante, se comprobó que el valor 1 no se encontraba en el intervalo de confianza de las correlaciones entre las diferentes escalas (Anderson y Gerbing, 1988) y que el índice de varianza extraída de cada factor superaba el cuadrado de las covarianzas entre cada par de factores (Fornell y Larcker, 1981). Los resultados obtenidos permitieron confirmar la existencia de validez discriminante.

La validez de contenido quedó garantizada al utilizarse en la investigación escalas cuya fiabilidad y validez habían sido comprobadas previamente por otros autores en estudios previos.

Respecto a la fiabilidad, los resultados obtenidos a partir del indicador alpha de Cronbach mostraron valores satisfactorios (superiores o iguales al valor recomendado de 0.7 ) (Churchill, 1979). Como medidas complementarias de análisis se calculó la fiabilidad compuesta y la varianza extraída, que en ambos casos fueron superiores a los mínimos recomendados ( 0.7 en el primer caso y de 0.5 en el segundo).

\subsection{Contraste de hipótesis}

A continuación, se procedió a estimar el modelo de ecuaciones estructurales que presentó un buen ajuste $(\mathrm{S}-\mathrm{B} \chi 2=114.3725$; df $=71$; $\mathrm{p}<0.01$; BBNFI= 0.917; BBNNFI= 0.957; $\mathrm{CFI}=0.966$; IFI= 0.967; $\mathrm{MFI}=0.900 ; \mathrm{RMSEA}=0.055)$. La figura 2 recoge el modelo final mostrando la información de los parámetros estimados que conducen a la aceptación de las hipótesis propuestas.

Figura 2

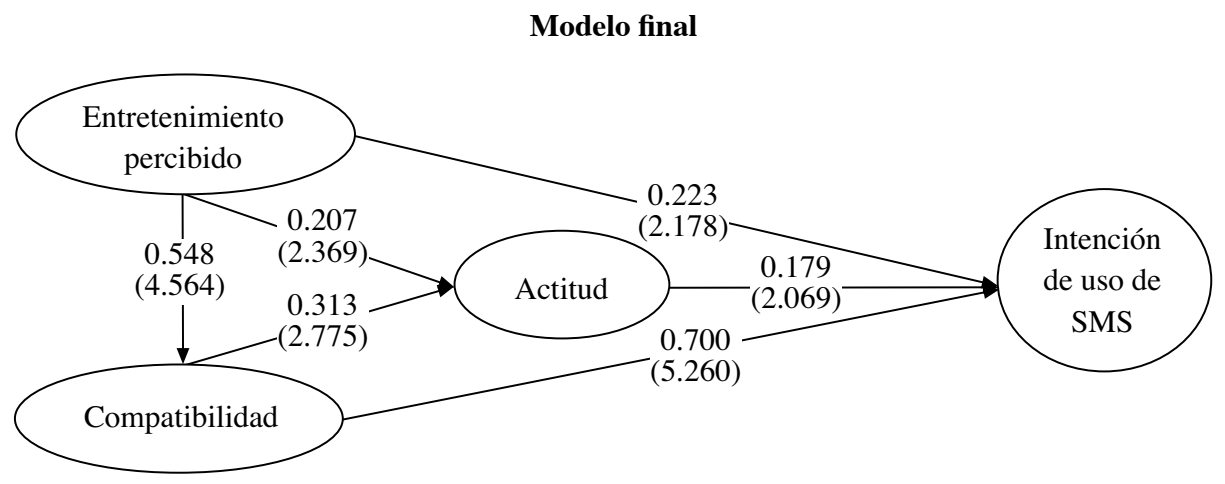

Del análisis de los resultados se desprende que la compatibilidad del individuo con el servicio de envío de SMS es la variable con mayor influencia en la intención de participar en los programas de televisión con SMS (H5 aceptada; $\beta=0.700 ; p<0.01$ ). El entretenimiento percibido que proporcionan los programas también tiene un efecto catalizador importante en la intención de participar en los programas de televisión con SMS (H6 aceptada; $\beta=0.223 ; \mathrm{p}<0.05$ ). Tanto el entretenimiento (H2 aceptada; $\beta=0.313 ; \mathrm{p}<0.01)$ como la compatibilidad ( $\mathrm{H} 3$ aceptada; $\beta=0.207 ; \mathrm{p}<0.01$ ) contribuyen a la formación de actitudes 
favorables al uso del servicio. El entretenimiento que proporcionan los programas televisivos influye positivamente sobre la compatibilidad individuo-móvil (H4 aceptada; $\beta=0.548$; $\mathrm{p}<0.01$ ), lo que confirma que las motivaciones intrínsecas son tan importantes como las extrínsecas para fomentar el envío de SMS para participar en los programas televisivos. Otro resultado importante es el papel que desempeña la actitud (H1 aceptada; $\beta=0.179$; $\mathrm{p}<0.05)$ como paso previo al comportamiento, ya que incide de forma directa y positiva sobre la intención de participar mediante SMS en los programas televisivos.

\section{CONCLUSIONES}

1. En los últimos años, las elevadas tasas de penetración y uso de los dispositivos móviles en la mayoría de países han favorecido el desarrollo de estrategias de marketing conducentes a incrementar la rentabilidad de los usuarios a través del consumo de servicios adicionales a los básicos de voz. Las estrategias publicitarias que incentivan que el público tome parte activa en la programación televisiva mediante el uso de los mensajes cortos a través del móvil están mostrando en estos últimos años una eficacia tal, que se hace necesaria la investigación en este ámbito.

2. Hasta el momento, los estudios sobre la interacción de los servicios móviles con otros medios de comunicación como la televisión han sido escasos y de carácter descriptivo, realizando esta investigación una contribución a la literatura académica al analizar los factores que contribuyen a la utilización de mensajes SMS para participar en programas televisivos, integrando en el modelo propuesto variables referidas a ambos medios de comunicación (televisión y móvil).

3. La edad del individuo resulta ser una variable clave en este tipo de estudios, ya que son los jóvenes no sólo los que más usan el teléfono móvil sino también los que en mayor proporción ven programas de entretenimiento.

4. Es el grupo de más jóvenes (de edad similar o por debajo a 24 años) el que utiliza en mayor medida el servicio de envío SMS, sustituyendo de ese modo a las llamadas de voz. Uno de los factores que contribuye a este resultado es el hecho de que no perciban ingresos o los mismos sean bajos.

5. El que los jóvenes estén más familiarizados con el servicio SMS, lleva a que lo utilicen en mayor medida no sólo para comunicarse con amigos, familiares y conocidos sino también para participar en programas televisivos. Sus distintos usos no plantean ningún problema para los más jóvenes, siendo además un servicio compatible con su forma de vida, estando dispuestos a seguir utilizándolo en el futuro.

6. Las variables sobre la relación individuo-medio (compatibilidad con el servicio y entretenimiento percibido del programa) son clave en el modelo propuesto, ya que ejercen una influencia directa y positiva sobre la intención de participar mediante SMS en los programas televisivos. El papel que desempeña la compatibilidad es muy importante, ya que es la variable que ejerce una mayor influencia sobre la intención de uso de SMS, seguida por el entretenimiento percibido del programa. Este resultado ratifica investigaciones previas en el ámbito de la compra por móvil (Wu y Wang, 2005) y destaca la importancia de que se tenga en cuenta la interacción del consumidor con el servicio en el diseño de los nuevos servicios del móvil. 
7. Además, tanto la compatibilidad (en primer lugar) como el entretenimiento refuerzan las actitudes favorables del individuo hacia este servicio lo que se traduce, de forma indirecta, en intención de uso. Ambos factores también están relacionados entre sí, ya que el entretenimiento ejerce una influencia directa y positiva sobre la compatibilidad, lo que permite concluir, que existe una relación muy estrecha entre el móvil y la persona, ya que cuanto más se disfruta con los programas, en mayor medida se considera que participar en los mismos encaja con los estilos y forma de vida de los consumidores.

8. La actitud es otra variable con influencia significativa en la intención de uso de SMS para participar en programas de televisión, aunque menor que la de las otras variables del modelo. Esta relación es coherente con el resultado de investigaciones previas en el ámbito del comercio electrónico por móvil que muestran una fuerte correlación positiva entre ambas variables (Nysveen et al., 2005a,b). Se constata así que los comportamientos de los consumidores son congruentes con sus actitudes (Andrades, 2005).

\section{IMPLICACIONES DE GESTIÓN}

Los resultados obtenidos permiten plantear un conjunto de implicaciones para la gestión de empresas:

1. Los operadores televisivos deben conseguir que el grupo de individuos de más de 24 años incremente su participación en los programas televisivos mediante envío de SMS. Ello puede conseguirse:

- a través de incentivos promocionales por enviar SMS, tales como dinero, regalos, participación en sorteos, etc,

- utilizando presentadores conocidos y con credibilidad que inciten a la participación, explicando paso por paso a los más mayores cómo se debe enviar el mensaje,

- dado que no es posible que todos los mensajes sean publicados en directo y ello puede ser motivo de no envío, se recomienda que los presentadores comenten constantemente en directo que los mensajes que no salen en pantalla salen publicados en la página web del programa, insistiendo en la elevada audiencia que tiene también la web,

- facilitando, cuando se pueda, las respuestas a los más mayores, brindando distintas alternativas de respuesta de la pregunta de la cuál se solicita participación, ya que en muchas ocasiones no se envían mensajes porque cuesta mucho escribir el texto o no se sabe expresar en poco espacio lo que se quiere, lo que a su vez reduce la compatibilidad percibida por el usuario.

2. Los operadores televisivos también deben incentivar el envío de SMS en programas que resulten entretenidos para el público objetivo al que se dirigen, ya que la influencia del entretenimiento percibido en la compatibilidad y actitud hacia el uso de los servicios por móvil ha puesto de manifiesto la necesidad de tener en cuenta la influencia de las motivaciones hedónicas en el uso del móvil para enviar SMS.

3. También recomendamos que en la publicidad sobre los programas en ambos medios (móvil y televisión), las empresas diseñen mensajes que además de informativos 
transmitan las posibles experiencias lúdicas que podrían derivarse de la participación, ya que de ese modo se incrementará la probabilidad de participar en los mismos.

4. Por último, señalar que las empresas de telefonía móvil deben incrementar la compatibilidad percibida y mejorar la actitud hacia el uso del móvil para enviar SMS, lo que pueden conseguir logrando una identificación del estilo de vida del consumidor con el uso del móvil para enviar SMS en los mensajes publicitarios. Además, deben cuidar mucho tanto el nivel estético como las funcionalidades que ofrecen los móviles. Una mayor personalización de los servicios del móvil también puede fortalecer la relación entre el dispositivo móvil y la persona que lo utiliza.

\section{LIMITACIONES Y FUTURAS LÍNEAS DE INVESTIGACIÓN}

La principal limitación del estudio radica en el uso de un muestreo de conveniencia que limita la generalización de los resultados obtenidos, aunque ha sido ampliamente utilizado en las investigaciones sobre el móvil (Wu y Wang, 2005; Yang, 2005). Otra posible limitación es que sólo se ha tenido en cuenta la compatibilidad y el entretenimiento percibido como variables vinculadas a la relación individuo-medio. Como futura línea de investigación proponemos considerar otros factores que pueden contribuir a la aceptación del servicio por parte del consumidor e incorporar los mismos al modelo propuesto. Dado que el presente artículo incorpora la actitud como variable mediadora en el modelo propuesto, otra posible línea de investigación consistiría en comparar el modelo propuesto con un modelo rival en el que la actitud actuara como variable moderadora.

\section{REFERENCIAS BIBLIOGRÁFICAS}

AJzen, I. (1991): “The Theory of Planned Behavior”, Organizational Behavior and Human Decision Processes, Vol. 50, No.2, pp. 179-211.

ANDERSON, J. y GERBING, D. (1988): "Structural equation modeling in practice: A review and recommended two-step approach", Psychological Bulletin, Vol. 103, № 3, pp. 411-423.

Anderson, J.A. y Meyer, T.P. (1975): "Functionalism and the mass media", Journal of Broadcasting, Vol. 19, $\mathrm{N}^{\circ} 1$, pp. 11-22.

AndRades, L. (2005): "Medición de las actitudes de los internautas respecto a la compra on-line. Segmentación en base a actitudes y caracterización de los segmentos identificados", Economic Analysis Working Paper,Vol. 4, No 4, pp. 1-85.

BABIN, B.; DARDEN, W. y GRIFFIN, M. (1994). "Work and/or fun: measuring hedonic and utilitarian shopping value", Journal of Consumer Research, Vol. 20, No 4, pp. 644-656.

BAGOZZI, R. Y YI, Y. (1988): "On the evaluation of structural equation models", Journal of the Academy of Marketing Science, Vol. 16, N 1, pp. 74-94.

BAGOZZI, R.P. y BURNKRANT, R.E. (1979): "Attitude organization and the attitude-behavior relationship", Journal of Personality and Social Psychology, $\mathrm{N}^{\circ}$ 37, p.6.

BAGOZZI, R.P. y BURNKRANT, R.E. (1985): "Attitude organization and the attitude-behavior relationship: a reply to Dillon and Kumar", Journal of Personality and Social Psychology, No 49, pp.1-16.

Baron, S.; PAtTerson, A. y Harris, K. (2006): "Beyond technology acceptance: understanding consumer practice”, International Journal of Service Industry Management, Vol.17, No.2, pp. 111135 . 
Beyer, Y.; Enli, G.S.; MaAso, A.J. y Ytreberg, E. (2007): "Small talk makes a big difference: Recent Developments in interactive, SMS-based television", Television \& New Media, Vol. 8, pp. 213-234.

Bigné, E.; AldÁs, J.; Ruiz, C. y SANZ, S. (2008): "Influencia de la compatibilidad, la propensión a innovar y el entretenimiento percibido en la decisión de compra a través del móvil”. Revista Portuguesa de Marketing, Vol.23, pp. 34-46.

Chaiken, S. y Stangor, C. (1987): "Attitudes and attitude change", Annual Review of Psychology, $\mathrm{N}^{\mathrm{o}} 38$, pp. $575-630$.

Chen, X. y Gongmin, B. (2007): “The Influence of Innovativeness Compatibility on Consumers' Online Purchase Intention on the Creative Product: A Case Study of Fashion”, IEEE, pp.6706-6709.

CheOng, J.H. y PARK, M. (2005): "Mobile internet acceptance in Korea", Internet Research, Vol. 15, No. 2, pp. 125-140.

Churchill, G.A. (1979): "Paradigm for Developing Better Measures of Marketing Constructs", Journal of Marketing Research, Vol. 16, № 1, pp. 64-73.

Comisión Del Mercado De Las Telecomunicaciones (2008). Informe trimestral Octubre-Diciembre 2008. Documento electrónico disponible en http://www.cmt.es (17 de Marzo de 2009).

DAvis, F. (1989): "Perceived usefulness, perceived ease of use and user acceptance of information technology", MIS Quaterly, Vol. 13, No.3, pp. 319-340.

Davis, F.; Bagozzi, R. y Warshaw, P. (1992): "Extrinsic and Intrinsic Motivation to Use Computers in the Workplace1", Journal of Applied Social Psychology, Vol. 22, N 14, p. 1132.

Davis, F.D.; BagozzI, R.P. y Warshaw, P.R. (1989): "User acceptance of computer technology: a comparison of two theoretical models", Management Science, Vol. 35, No.8, pp. 33-47.

FishbeIn, M.y AJZen, I. (1975). Belief, attitude, intention and behavior: an introduction to theory and research. Nueva York, Addison-Wesley.

ForNELL, C. y LARCKER, D. (1981): "Evaluating structural equation models with unobservable variables and measurement error", Journal of Marketing Research, Vol. 18, № 1, pp. 39-50.

GEORGE, J.F. (2004): "The theory of planned behavior and Internet purchasing", Internet Research, Vol. 14, No. 3, pp. 198-212.

HA, I.; Yoon, Y. y CHOI, M. (2007): "Determinants of adoption of mobile games under mobile broadband wireless access environment", Information \& Management, Vol. 44, pp. 276-286.

HATCHER, L. (1994). A Step-by-Step approach to using the SAS system for factor analysis and structural equation modelling. Cary, NC, SAS Institute Inc.

Herring, S. C. y ZelenkausKaite, A. (2009): "Symbolic capital in a virtual heterosexual market: Abbreviation and insertion in Italian iTV SMS", Written Communication, Vol. 26, No. 1. Documento electrónico disponible en de Febrero http://ella.slis.indiana.edu/ herring/wc.2009.pdf (5 de Febrero de 2009).

Hsu, C.L.; Lu, H.P. y Hsu, H.H. (2007): "Adoption of the mobile Internet: An empirical study of multimedia message service (MMS)", Omega, Vol. 35, No. 6, pp.715-726.

Jöreskog, K. y Sorbom, D. (1990). LISREL 7. A Guide to Program and Application. Michigan, SPSS.

Katz, E.; Blumler, J. y Gurevitch, M. (1974): "Utilization of mass communication by the individual", en Blumler, J. y Katz, E. (Eds.), The uses of mass communications: Current perspectives on gratifications research, (pp. 19-32). Beverly Hills, CA, Sage.

Kuo, Y. y Yen, S. (2009): "Towards an understanding of the behavioural intention to use 3G mobile value-added services", Computers in Human Behaviour, Vol. 25, pp. 103-110.

LAforet, S. y Li, X. (2005): "Consumers'attitudes towards online and mobile banking in China", International Journal of Bank Marketing, Vol. 23, №5, pp. 362-380.

LEUNG, L. Y WeI, R. (2000): "More than just talk on the move: Uses and gratifications of the cellular phone", Journalism and Mass Communication Quarterly, Vol. 77, № 2, p. 308. 
Liao, C; Tsou, C. y Huang, M. (2007): "Factors influencing the usage of 3G mobile services in Taiwan”, Online Information Review, Vol. 31, No. 6, pp.759-774.

Lu, Y.; TAO, Z. Y WANG, B. (2009): “Exploring Chinese users' acceptance of instant messaging using the theory of planned behavior, the technology acceptance model and the flow theory", Computers in Human Behaviour, Vol. 25, pp. 29-39.

Mallat, N.; Rossi, M.; Tuunainen, V.y Öörni, A. (2009): The impact of use context on mobile services acceptance: The case of mobile ticketing, Information \& Management, Vol. 46, pp. 190-195.

McGuire, W.J. (1985): “Attitudes and attitude change”, en Lindzey G. y Aronson, E. (Eds.), Handbook of Social Psychology, 19 (pp. 233-346). New York, Random House.

McQuail, D. (1995). Mass communication theory (tercera edición). California: Sage.

Moore, G. y Benbasat, I. (1991): "Development of an Instrument to Measure the Perceptions of Adopting an Information Technology Innovation", Information Systems Research, Vol. 2, No 3, pp. 192-222.

MunnukKa, J. (2007): "Characteristics of early adopters in mobile communications markets", Marketing Intelligence \& Planning, Vol. 25, No. 7, pp. 719-731.

Netsize (2010). The Netsize guide 2010. Documento electrónico disponible en http://www.netsize. com/Download/Guide/TheNetsizeGuide2010.pdf (8 de Mayo de 2010).

Nysveen, H.; Pedersen, P.E. y ThorbJornsen, H. (2005a): "Explaining intention to use mobile chat services: moderating effects of gender", Journal of Consumer Marketing, Vol. 22, No.5, pp. 247-256.

Nysveen, H.; Pedersen, P.E. y Thorbjornsen, H. (2005b): "Intentions to Use Mobile Services: Antecedents and Cross-Service Comparisons", Academy of Marketing Science Journal, Vol. 33, No.3, pp. 330-346.

Rogers, E. (1995). Diffusion of Innovations (cuarta edición). New York, The Free Press.

TAYLOR, S. Y TODD, P.A. (1995): "Understanding information technology usage: A test of competing models", Information Systems Research, Vol. 6 No. 2, pp.144-176.

ViJaYASARATHY, L.R. (2004): "Predicting consumer intentions to use on-line shopping: the case for an augmented technology acceptance model", Information \& Management, Vol. 41, $\mathrm{N}^{\circ}$ 6, pp. $747-762$.

Weiss, H.M. y Cropanzano, R. (1996): "Affective events theory: a theoretical discussion of the structure, causes and consequences of affective experiences at work", en Staw B.M. y Cummings, L.L. (Eds.), Research in Organizational Behavior, (pp. 1-74). Greenwich, CT, JAI Press.

WU, J.H. Y WANG, S.C. (2005): "What drives mobile commerce? An empirical evaluation of the revised technology acceptance model", Information \& Management, Vol. 42, No.5, pp. 719-729.

YANG, K.C. (2005): "Exploring factors affecting the adoption of mobile commerce in Singapore", Telematics and Informatics, Vol. 22, No. 3, pp. 257-77. 